\section{Synthesis of Sparse Arrays With Focused or Shaped Beampattern via Sequential Convex Optimizations}

\author{
Benjamin Fuchs
}

\begin{abstract}
An iterative procedure for the synthesis of sparse arrays radiating focused or shaped beampattern is presented. The algorithm consists in solving a sequence of weighted $l_{1}$ convex optimization problems. The method can thus be readily implemented and efficiently solved. In the optimization procedure, the objective is the minimization of the number of radiating elements and the constraints correspond to the pattern requirements. The method can be applied to synthesize either focused or shaped beampattern and there is no restriction regarding the array geometry and individual element patterns. Numerical comparisons with standard benchmark problems assess the efficiency of the proposed approach, whose computation time is several orders of magnitude below those of so-called global optimization algorithms.
\end{abstract}

Index Terms-Array pattern synthesis, convex programming, linear arrays, planar arrays, sparsity.

\section{INTRODUCTION}

The synthesis of antenna arrays with a minimum number of elements has several practical advantages such as the reduction of weight, cost, power consumption and the simplification of the feeding network. Depending on the synthesis method, such arrays are called aperiodic, non redundant, random, thinned, spaced tapered or sparse.

Generally the reduction of the number of antenna elements calls for the design of non uniformly spaced arrays. Many techniques have been proposed over the last fifty years to synthesize such arrays [1]. One can mention spatial tapering designs [2], [3], stochastic methods (genetic algorithm [4], particle swarm [5], ant colony [6], simulated annealing [7], [8]), combinatorial approaches [9], [10] and various mathematical programming methods such as linear programming [11], [12]. Matrix pencil methods (MPM) have lately been efficiently applied to reconstruct focused and shaped beampatterns while reducing the number of the array elements [13]-[15].

It is only recently that a few methods exploiting the partial convexity of sparse antenna array synthesis problems have been proposed. A hybrid approach, combining a global optimization method (simulated annealing or genetic algorithm) to determine the element locations and convex programming to find the element excitations, has thus been efficiently applied to the synthesis of sparse linear [16] and planar [17] arrays. In [18], a procedure to synthesize sparse arrays with antenna selected via convex optimization is presented. The iterative method uses conjugate symmetric excitations and allows to synthesize only symmetric shaped beampatterns in order to keep the convexity of the problem.

The problem addressed in this communication is the synthesis of arrays, having as few elements as possible, whose radiation pattern can

Manuscript received August 25, 2011; revised November 21, 2011; accepted January 24, 2012. Date of publication May 01, 2012; date of current version July 02, 2012.

The author is with the Laboratory of Electromagnetics and Acoustics, Ecole Polytechnique Fédérale de Lausanne, Switzerland on leave from the Institute of Electronics and Telecommunications of Rennes (IETR), University of Rennes 1, France (e-mail: b.fuchs@epfl.ch).

Color versions of one or more of the figures in this communication are available online at http://ieeexplore.ieee.org.

Digital Object Identifier 10.1109/TAP.2012.2196951 be either a focused beam or any given far field. The method can be applied to arbitrary arrays, i.e., arrays of any geometry and composed of elements having potentially differing radiation patterns.

To synthesize sparse arrays, an iterative algorithm, introduced in [19], [20], is applied. It consists in solving a sequence of reweighted convex $l_{1}$ minimization problems.

The communication is organized as follows. In Section II, the sparse array synthesis problem is described and the proposed resolution method is detailed. To show its efficiency, the approach is applied in Section III to solve standard sparse array synthesis problems. Conclusions are drawn in Section IV.

\section{Problem Formulation AND Resolution}

Let us consider an array composed of $N$ elements placed at arbitrary but known locations $\vec{r}_{i}$ with $i=1, \ldots, N$. For the sake of clarity, the problem is described for a one-dimensional pattern synthesis. The synthesis is performed over the polar angle $\theta$ in a fixed azimuthal plane $\varphi=\varphi_{0}$ that is omitted in the notations. The extension to a two-dimensional (2-D) pattern synthesis, i.e., a synthesis over both angular directions $\theta$ and $\varphi$, is straightforward and examples of 2-D pattern synthesis are shown in Section III. Each element $i$ radiates a pattern $g_{i}(\theta)$ in the direction $\theta$. The far field $f$ radiated by the array is then

$$
f(\theta)=\sum_{i=1}^{N} a_{i}(\theta) w_{i}, \quad \text { with } a_{i}(\theta)=g_{i}(\theta) e^{j(2 \pi / \lambda) \overrightarrow{r_{i}} \cdot \vec{r}(\theta)}
$$

where $w_{i}$ is the excitation of the $i$-th element and $\vec{r}(\theta)$ is the unit vector in the direction $\theta$.

Introducing the $N$-dimensional (with $N$ large) steering and excitation vectors, $\boldsymbol{a}(\theta)$ and $\boldsymbol{w}$ respectively, (1) is rewritten

$$
f(\theta)=\boldsymbol{a}(\theta)^{T} \boldsymbol{w}
$$

where.$^{T}$ denotes the transpose operator.

The synthesis problem addressed amounts to find an array, with as many zero components among the $N$ in $\boldsymbol{w}$ as possible, that complies to pattern constraints.

\section{A. Array Pattern Constraints}

1) Focused Beampattern: A focused beampattern (F.B.) is synthesized by applying the following constraints: a main beam radiated in the direction $\theta_{0}$ with sidelobes below a given upper bound $\rho(\theta)$ over an angular region $S$. These constraints can be written

$$
\text { (F.B.) }\left\{\begin{array}{l}
\Re\left\{f\left(\theta_{0}\right)\right\}=1 \\
|f(\theta)| \leq \rho(\theta), \quad \forall \theta \in S
\end{array} .\right.
$$

With the notations introduced in (2), these constraints become

$$
\text { (F.B.) }\left\{\begin{array}{l}
\Re\left\{\boldsymbol{a}\left(\theta_{0}\right)^{T} \boldsymbol{w}\right\}=1 \\
\left|\boldsymbol{a}(\theta)^{T} \boldsymbol{w}\right| \leq \rho(\theta), \quad \forall \theta \in S
\end{array}\right. \text {. }
$$

Note that enforcing the real part, instead of the magnitude, of the far field to be equal to one in the direction $\left(\theta_{0}\right)$ is not a limitation. The excitations $\boldsymbol{w}$ are indeed determined up to a phase and, by contradiction, it is easy to show that, at the optimum of the problem introduced below, see (8), the imaginary part of the field at $\theta_{0}$ will be zero and hence the amplitude equal to one as desired.

2) Shaped Beampattern: To synthesize a desired far field $f_{d}$ of any shape, one can impose

$$
\left|f(\theta)-f_{d}(\theta)\right| \leq \epsilon
$$


where the parameter $\epsilon$ is the degree of accuracy with which $f_{d}$ is approximated.

The constraint (5) can be applied either over the whole space or only in the main beam region $P$. In the latter case, which is the most practically encountered one, the shaped beam constraints (S.B.) can be written

$$
\text { (S.B.) }\left\{\begin{array}{ll}
\left|\boldsymbol{a}(\theta)^{T} \boldsymbol{w}-f_{d}(\theta)\right| \leq \epsilon, & \forall \theta \in P \\
\left|\boldsymbol{a}(\theta)^{T} \boldsymbol{w}\right| \leq \rho(\theta), & \forall \theta \in S
\end{array}\right. \text {. }
$$

\section{B. Sparse Array Synthesis}

Whereas the array pattern is constrained by applying either (4) or (6) depending on the targeted type of pattern, the sparsity of the array is the optimization goal of the synthesis problem. The sparse array synthesis problem can then be written

$$
\min _{\boldsymbol{w}}\|\boldsymbol{w}\|_{0} \text { under (F.B.) or (S.B.) }
$$

where $\|\boldsymbol{w}\|_{0}$ denotes the number of non-zero components of $\boldsymbol{w}$.

The optimization problem (7) is non convex and generally impossible to solve, since finding its solution would require an intractable combinatorial search, even for moderate $N$. A common alternative is to consider the following convex problem

$$
\min _{\boldsymbol{w}}\|\boldsymbol{w}\|_{1} \text { under (F.B.) or (S.B.) }
$$

where $\|\boldsymbol{w}\|_{1}=\sum_{i=1}^{N}\left|w_{i}\right|$. The use of the $l_{1}$ norm is indeed known to produce sparse solutions for a wide range of applications [21], [22].

To further enhance the sparsity of the solution $\boldsymbol{w}$ of the problem (8), an algorithm that consists in solving a sequence of weighted $l_{1}$ minimization problems has been recently proposed by [19], [20]. The idea of this algorithm is to bring the weighted $l_{1}$ criterion as close as possible to the $l_{0}$ criterion (7). For the $l_{1}$ norm, larger coefficients are indeed penalized more heavily than smaller coefficients, whereas the $l_{0}$ norm penalizes uniformly all non-zero coefficients.

The weighted $l_{1}$ minimization problem solved at the iteration $k$ is

$$
\begin{aligned}
& \min _{\boldsymbol{w}^{k}} \sum_{i=1}^{N} \alpha_{i}{ }^{k}\left|w_{i}{ }^{k}\right| \text { under (F.B.) or (S.B.), } \\
& \text { where } \alpha_{i}{ }^{k}=\left(\left|w_{i}{ }^{k-1}\right|+\epsilon\right)^{-1} \text { with } \epsilon>0 .
\end{aligned}
$$

The positive weights $\alpha_{i}{ }^{k}$ are computed from the values of the previous solution $w_{i}^{k-1}$. With this definition, it happens that small magnitude excitations $\left|w_{i}\right|$ lead to large weights $\left(\left|w_{i}\right|+\epsilon\right)^{-1}$ and are thus penalized at the next iteration. Conversely, large excitations give small weights that enforce their reconduction at the next iteration. The parameter $\epsilon>0$ provides numerical stability and ensures that a zero excitation $w_{i}{ }^{k}$ at step $k$ does not strictly prohibit to have $w_{i}^{k+1} \neq 0$ at step $k+1$. Although the algorithm is reasonably robust to the choice of the parameter $\epsilon$, it has been empirically shown in [20] that $\epsilon$ should be set slightly smaller than the smallest non-zero magnitude one is willing to implement.

The iterative algorithm is as follows.

- In the first iteration $(k=1)$, find a $\boldsymbol{w}^{1}$ that satisfy the constraints (4) or (6), an admissible point.

- Set the parameter $\epsilon>0$ (see above).

1) For $k>1$, solve the convex minimization problem (9).

2) Update the weights: $\alpha_{i}^{k}=\left(\left|w_{i}^{k-1}\right|+\epsilon\right)^{-1}$ for $i=1, \ldots, N$.

3) Repeat steps 1 and 2 until $\left\|\boldsymbol{w}^{k+2}\right\|_{0}=\left\|\boldsymbol{w}^{k}\right\|_{0}$, i.e., until the number of active elements does no longer change three times in a row.

As a starting point, a large number $N$ of elements are distributed over the given array aperture, i.e., an array with small inter-element spacings is considered. This number $N$ can be a priori reduced in some specific synthesis cases, for instance when the pattern to be synthesized is symmetrical. It is important to note that $N$ must be sufficiently large in order to lead to a true sparse array and not simply to a thinned one but not too large in order to keep the numerical efficiency of the algorithm. A reasonable trade-off, applied in Section III, is to choose an inter-element spacing of $0.01 \lambda$ and $0.25 \lambda$ for 1-D and 2-D pattern synthesis respectively.

Then by solving at each iteration a convex optimization problem, an increasing number of array elements are set to zero. Although it has been experimentally noticed that the algorithm converges after only a few iterations, the optimality of the solution can not be guaranteed.

\section{Resolution}

To solve the sparse array synthesis problem (9), the constraints are first discretized by introducing $L$ directions $\left\{\theta_{l}\right\}, l=1, \ldots, L$ that cover $P \cup S$. These directions are divided into two subsets of $K$ and $M$ directions associated with $P$ and $S$ respectively. One then defines a $K \times N$ matrix $\boldsymbol{A}^{K}$ and a $M \times N$ matrix $\boldsymbol{A}^{M}$ whose $k$-th and $m$-th row are $\boldsymbol{a}\left(\theta_{k}\right)^{T}$ and $\boldsymbol{a}\left(\theta_{m}\right)^{T}$, respectively. Moreover, let $\boldsymbol{\rho}^{M}$ and $\boldsymbol{f}_{d}{ }^{K}$ be $M$ and $K$ dimensional real vectors associated with $\rho$ and $f_{d}$.

The constraints (4) and (6) become

$$
\text { (F.B.) }\left\{\begin{array}{l}
\Re\left\{\boldsymbol{a}_{0}{ }^{T} \boldsymbol{w}\right\}=1, \text { with } \boldsymbol{a}_{0}=\boldsymbol{a}\left(\theta_{0}\right) \\
\left|\boldsymbol{A}^{M} \boldsymbol{w}\right| \leq \boldsymbol{\rho}^{M}
\end{array}\right.
$$

and

$$
\text { (S.B.) }\left\{\begin{array}{l}
\left|\boldsymbol{A}^{K} \boldsymbol{w}-\boldsymbol{f}_{d}^{K}\right| \leq \epsilon \mathbf{1}^{K} \\
\left|\boldsymbol{A}^{M} \boldsymbol{w}\right| \leq \boldsymbol{\rho}^{M}
\end{array}\right.
$$

respectively, where $\mathbf{1}^{K}$ is column vector of ones of dimension $K$ and $\left|\boldsymbol{x}^{U}\right| \leq \boldsymbol{\delta}^{U}$ is equivalent to $\left|x_{i}\right| \leq \delta_{i}$, for $i=1, \ldots, U$.

The optimization problem (9) is then transformed into a second order cone program (SOCP), whose standard form is

$$
\min _{\boldsymbol{x}} \boldsymbol{c}^{T} \boldsymbol{x}, \text { under } \boldsymbol{B} \boldsymbol{x}=\boldsymbol{b}, \boldsymbol{x} \in K
$$

where $K$ represents second order cones. A second order cone $L_{p}$ of dimension $p$ is of the form

$$
\begin{aligned}
L_{p} & =\left\{x \mid x_{1} \geq\left\|x_{2}, x_{3}, \ldots, x_{p}\right\|_{2}\right\} \\
& =\left\{\boldsymbol{x} \mid x_{1} \geq\left(\sum_{i=2}^{p} x_{i}^{2}\right)^{1 / 2}\right\} .
\end{aligned}
$$

A SOCP can be seen as a generalization of a linear program. It has been established in [23] that one can extend the linear program (theory and algorithms) to the conic one.

The translation of the convex optimization problem (9) into a SOCP (12) requires some transformations. The inequality constraints in (10) and (11) are of the form $\left|x_{i}\right| \leq \delta_{i}$ for $i=1, \ldots, U$. To be transformed into equalities, as required in (12), one associates to each complex $x_{i}$ a triplet of real variables $\left\{t_{i}, \Re\left(x_{i}\right), \Im\left(x_{i}\right)\right\} \in L_{3}$ for $i=1, \ldots, U$, which is such that $t_{i} \geq\left|x_{i}\right|=\left[\Re\left(x_{i}\right)^{2}+\Im\left(x_{i}\right)^{2}\right]^{1 / 2}$, hence $\left|x_{i}\right| \leq$ $t_{i} \leq \delta_{i}$. One then introduces $U$ real and positive variables $v_{i}$ (called slack variables) to get the equalities: $v_{i}+t_{i}=\delta_{i}$, for $i=1, \ldots, U$.

The cost function $\min _{w} \sum_{i=1}^{N} \alpha_{i}\left|w_{i}\right|$ in (9) is put in the form $\boldsymbol{c}^{T} \boldsymbol{x}$ by simply inserting the $\alpha_{i}$ 's in the right positions in $\boldsymbol{c}^{T}$.

Once the transcription of the synthesis problem into a SOCP has been performed, there are many readily available software that solve efficiently SOCP in roughly the same time as a linear program of equivalent size and without any specific tuning. The optimization toolbox SeDuMi [24] is used in this work. 


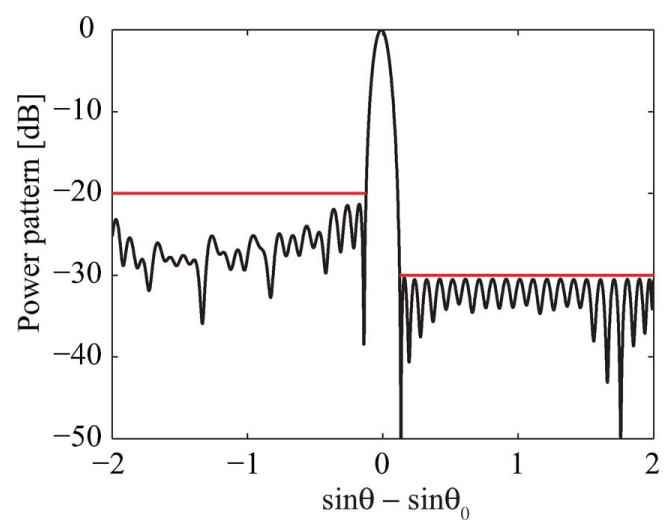

Fig. 1. Synthesized linear sparse array of 22 elements with an aperture of $9.66 \lambda$. The far field constraints are asymmetric. The sidelobes are below $-30.3 \mathrm{~dB}$ and $-20.3 \mathrm{~dB}$ for $\left|\sin \theta-\sin \theta_{0}\right|>0.12$.

\section{NUMERICAL APPLICATIONS}

In this Section, numerical applications of sparse array synthesis with first focused and then shaped beampatterns are presented. Several benchmark problems of linear and planar sparse array synthesis are considered to assess the efficiency of the proposed approach.

\section{A. Focused Beam Synthesis}

1) Sparse Linear Array: The synthesis of a linear array of maximum length $10 \lambda$ whose pattern is subject to asymmetric constraints, namely a $10 \mathrm{~dB}$ difference between the sidelobe levels on each side of the main beam, has been addressed by [16], [25]. The constraints on the sidelobe are enforced for the directions $\theta$ such as $0.12 \leq\left|\sin \theta-\sin \theta_{0}\right| \leq 2$, where $\theta_{0}$ is the direction of the main beam. This means that the main beam can be steered without any grating lobes between the two endfire directions $\theta_{0}= \pm \pi / 2$.

Up to now, the best results reported in the literature have been obtained using the hybrid approach proposed in [16]. With a linear array composed of 21 elements and of length $9.7 \lambda$, the synthesized pattern achieves sidelobes of maximum levels $-29.8 \mathrm{~dB}$ and $-19.8 \mathrm{~dB}$.

Using the proposed algorithm, a linear array of length $9.66 \lambda$ and composed of 22 elements has been synthesized. Sidelobes below $-30.3 \mathrm{~dB}$ and $-21.3 \mathrm{~dB}$ are achieved, as shown in Fig. 1. The optimized element locations and associated complex excitations are given in Table I.

2) Sparse Planar Array: Let us now consider the problem of synthesizing a sparse planar array of elements located in a square of side 5 $\lambda$ in order to radiate a pattern having a broadside main beam of width $\sin \theta_{s}=0.239$ at $-6 \mathrm{~dB}$.

With a hybrid approach combining simulated annealing and convex programming [17], sidelobes below $-16.5 \mathrm{~dB}$ are achieved with a planar array composed of only 41 elements.

By applying the proposed algorithm, a main beam width of 0.240 at $-6 \mathrm{~dB}$ and a maximum sidelobe level of $-17.3 \mathrm{~dB}$ are synthesized with a planar array of 41 elements in only ten iterations taking less than four minutes. The synthesized radiation patterns and array layout are shown in Fig. 2, where one reminds that $\theta$ and $\varphi$ are the polar and azimuthal angles respectively.

\section{B. Shaped Beam Synthesis}

For the following shaped beam synthesis problems, the constraints (6) are applied with $\epsilon=10^{-2}$.

1) Sparse Linear Arrays:

a) Flat-Top Pattern: The goal is to synthesize a sparse linear array of maximum length $8 \lambda$ to radiate a sectoral pattern. The desired pattern
TABLE I

SYNTHESIZED LOCATIONS AND EXCITATIONS OF THE LINEAR ARRAY SUBJECT TO ASYMMETRIC CONSTRAINTS

\begin{tabular}{cccc}
\hline Element number & Locations & \multicolumn{2}{c}{ Excitations } \\
$i$ & $\| \overrightarrow{r_{i} \| \text { in }[\lambda]}$ & $\left|w_{i}\right|$ & $\angle w_{i}[\mathrm{deg}]$ \\
\hline 1 & 0 & 0.866 & 7.85 \\
2 & 0.34 & 0.678 & 46.35 \\
3 & 0.80 & 0.751 & 48.39 \\
4 & 1.27 & 0.859 & 49.63 \\
5 & 1.74 & 0.983 & 49.03 \\
6 & 2.20 & 1.105 & 51.24 \\
7 & 2.67 & 1.205 & 51.00 \\
8 & 3.14 & 1.319 & 50.47 \\
9 & 3.60 & 1.409 & 53.87 \\
10 & 4.07 & 1.457 & 53.60 \\
11 & 4.53 & 1.463 & 57.63 \\
12 & 5.00 & 1.422 & 57.39 \\
13 & 5.47 & 1.395 & 57.38 \\
14 & 5.93 & 1.312 & 61.65 \\
15 & 6.40 & 1.200 & 62.02 \\
16 & 6.86 & 1.061 & 65.70 \\
17 & 7.33 & 0.903 & 65.97 \\
18 & 7.80 & 0.758 & 66.49 \\
19 & 8.26 & 0.633 & 69.77 \\
20 & 8.73 & 0.512 & 72.05 \\
21 & 9.20 & 0.381 & 79.23 \\
22 & 9.66 & 0.263 & 94.46 \\
\hline \hline
\end{tabular}

$\left(f_{d}\right.$ in (6)) has been synthesized in [26] with an uniformly spaced array of 15 elements. This problem has been recently considered in [15] using a forward backward matrix pencil method (FBMPM) in which the desired pattern is discretized over the whole space including the sidelobe region. As shown in Fig. 3(b),(c), the results of the FBMPM are similar to those obtained applying our method, where only the shaped beam is discretized and the sidelobes are upper bounded, as formulated in (11). A sparse array composed of only 10 elements that radiates a shaped beam very close to the one desired is synthesized, see Fig. 3(a). This means that 5 elements are saved compared to the uniformly spaced array synthesized by [26].

b) Cosecant Pattern: The synthesis of a sparse linear array of maximum length $8 \lambda$ to radiate a non-symmetrical shaped beampattern is addressed. The desired pattern is a cosecant shaped beam obtained in [27] with an uniformly spaced array of 16 elements. By discretizing the shaped beam and constraining appropriately the sidelobes, the desired far field pattern is synthesized, see Fig. 4(a), with an array of only 13 elements. The complex weightings and element positions are compared to those found by [14] with the FBMPM in Fig. 4(b),(c).

2) Sparse Planar Arrays: The synthesis of a planar array of elements located in a square of side $5 \lambda$ that radiates a flat-top pattern having a circular symmetry is considered. The desired pattern $f_{d}$ is obtained with a planar array composed of $11 \times 11$ elements that are half wavelength spaced $\left(d_{x}=d_{y}=\lambda / 2\right)$ using a Kaiser window. The procedure, detailed in [28], is summarized as follows:

$$
\begin{aligned}
f_{d}(u, v) & =\sum_{m=-5}^{5} \sum_{n=-5}^{5} a_{m, n} w_{m, n} e^{j k\left(d_{x} u+d_{y} v\right)}, \\
\text { with } u & =\sin \theta \cos \varphi \text { and } v=\sin \theta \sin \varphi \\
a_{m, n} & =\psi_{R} \frac{J_{1}\left(\psi_{R} \sqrt{m^{2}+n^{2}}\right)}{2 \pi \sqrt{m^{2}+n^{2}}} \\
w_{m, n} & =\frac{I_{0}\left(\beta \sqrt{1-\frac{m^{2}+n^{2}}{\sqrt{20}}}\right)}{I_{0}(\beta)}
\end{aligned}
$$

where $J_{1}$ and $I_{0}$ are the first order Bessel function and the zero order modified Bessel function respectively. The parameter $\beta$ specifies the 


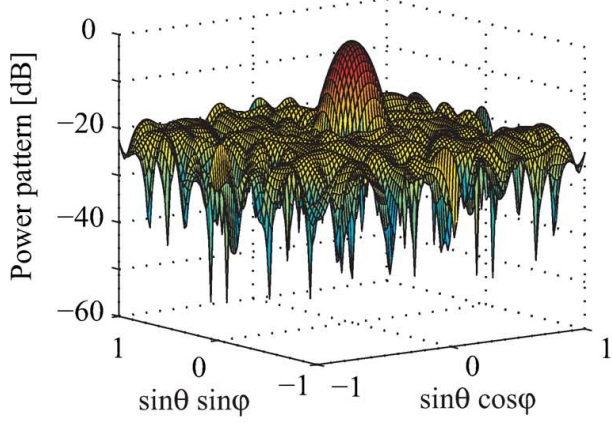

(a)

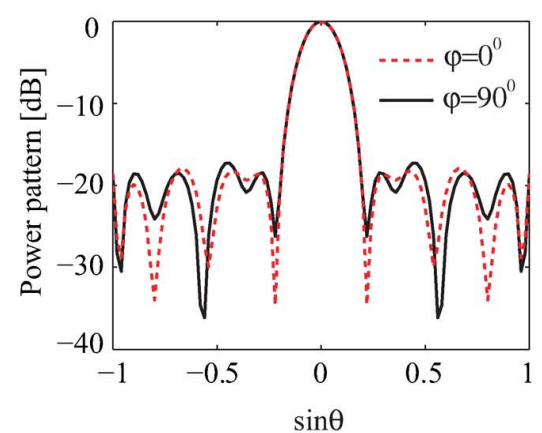

(b)

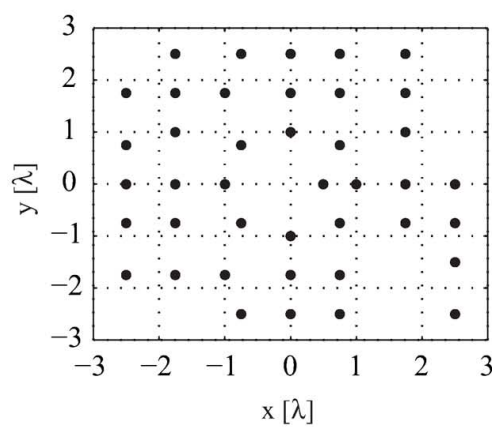

(c)

Fig. 2. Sparse planar array synthesis results: (a) 3D view of the far field pattern with (b) two $\varphi$-cutting planes and (c) optimized layout of the 41 element array.

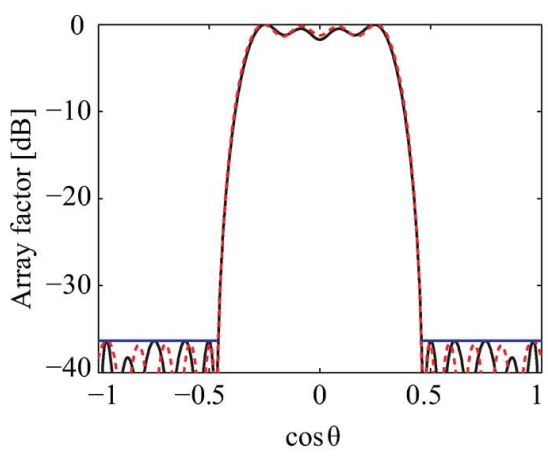

(a)

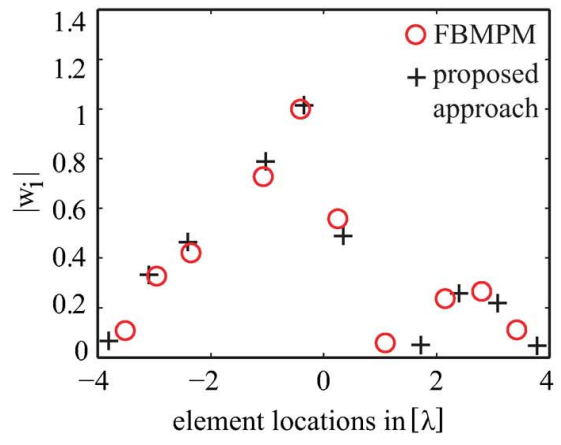

(b)

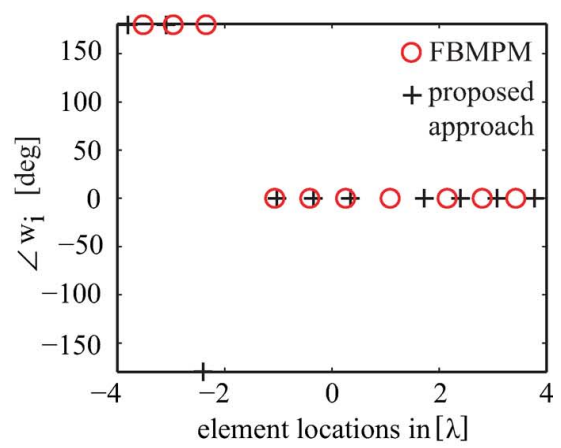

(c)

Fig. 3. (a) Reconstruction of a flat-top pattern given by [26] (dotted line) using the proposed approach (solid line). In (b),(c), the complex weightings of the synthesized 10 element array are compared to those obtained by FBMPM [15].

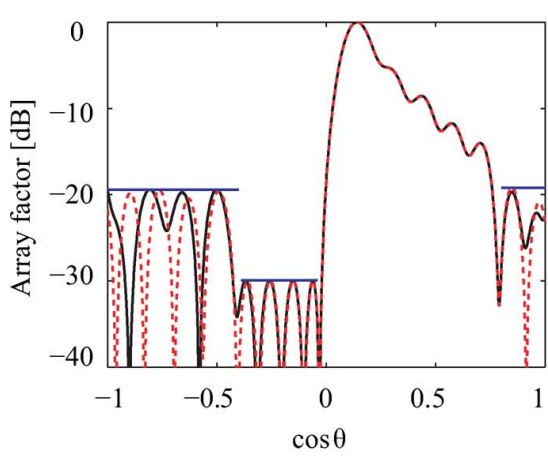

(a)

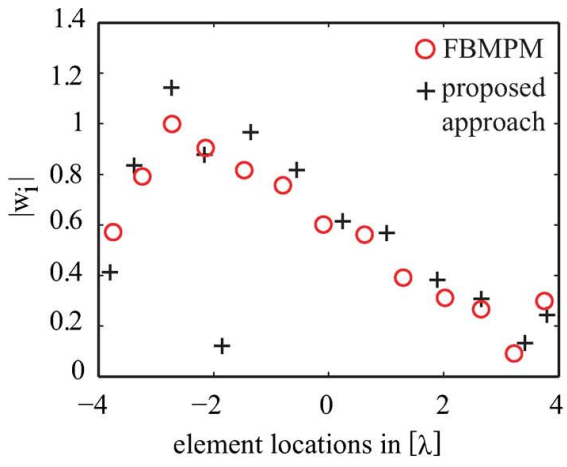

(b)

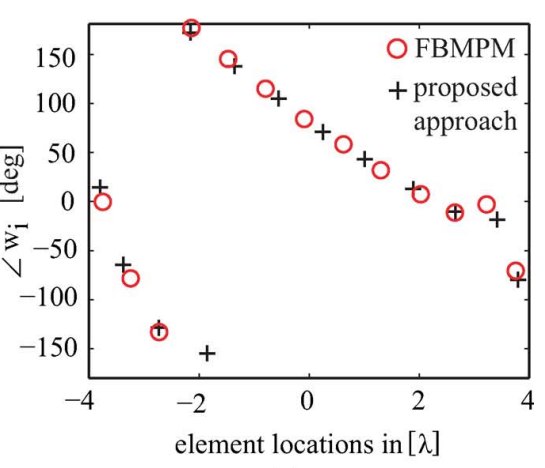

(c)

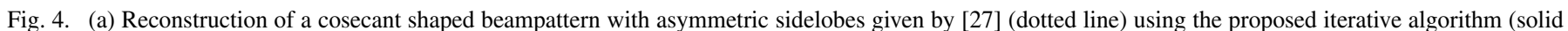
line). In (b),(c), the complex weightings of the synthesized 13 element array are compared to those obtained by FBMPM [14].

trade-off between the sidelobe level and the main beam width is equal to 5 and $\psi_{R}=0.4 \pi$.

An initial array composed of $21 \times 21$ elements $\left(d_{x}=d_{y}=\lambda / 4\right)$ is considered as a starting point to synthesize the desired shaped beampattern. Applying the proposed algorithm, this array is sparsified after a few iterations yielding to a only 100 element array whose layout is shown in Fig. 5(c). The synthesized far field patterns are given in Fig. 5(a),(b). The desired and synthesized patterns are superimposed in the shaped beam region whereas the sidelobes are below the required level. While keeping the same radiation performances and with the same radiation aperture, the proposed sparse array synthesis procedure allows to save 21 antennas, specifically $17 \%$ of the antennas of the uniform planar array are not used.

\section{CONCLUSION}

An iterative method to synthesize sparse arrays with focused or shaped beampattern has been presented. The goal is to minimize the number of elements of an array that radiates a pattern having either a main beam in a predefined direction and sidelobes below a given arbitrary envelope or any given far field.

Each iteration of the algorithm simply requires solving a convex optimization problem, namely a constrained weighted $l_{1}$ minimization problem [19], [20]. The synthesis problem is simple to implement and it can be solved efficiently using freely available subroutines. While the optimality of the solution is not guaranteed, the algorithm typically converges after a small number of iterations. To show its efficiency, 


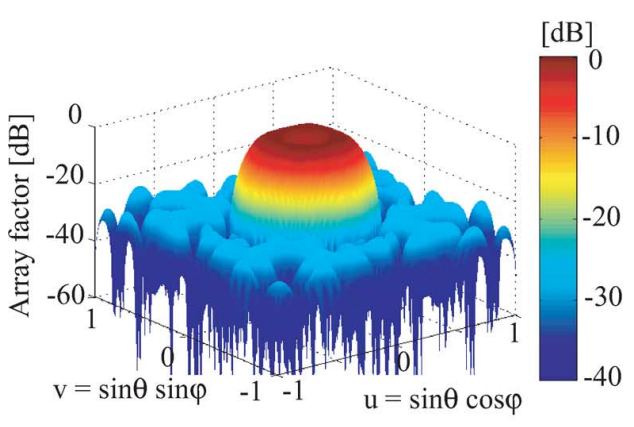

(a)

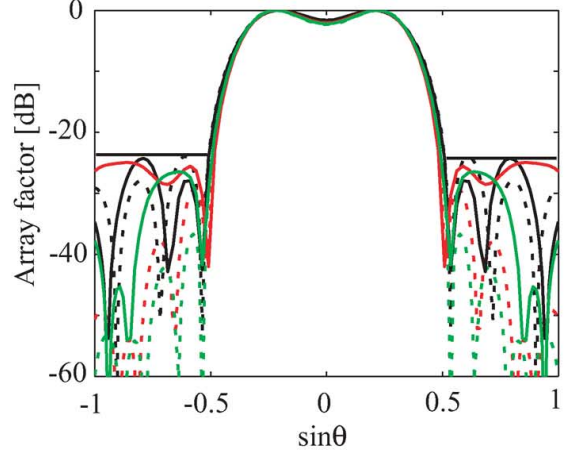

(b)

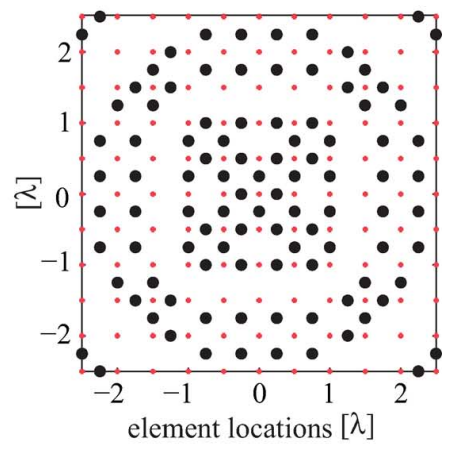

(c)

Fig. 5. Sparse shaped beam planar array synthesis results. (a) 3D view of the flat top pattern. (b) Beampattern cuts at $\Phi=0^{\circ}, 30^{\circ}$ and $45^{\circ}$ of the desired pattern (dotted line) and the synthesized one (solid line). (c) Optimized layout of the 100 element array (•) and element locations of the uniform array (.).

the proposed approach has been applied to solve standard benchmark problems. The synthesized sparse arrays compare favorably to the corresponding array designs found in the literature.

The advantages of the proposed algorithm over competing methods are manifold. First, its computation time is several orders of magnitude smaller than procedures involving stochastic methods. Second, there is no parameter to be tuned (fixed parameter $\epsilon$ and easy stopping criterion) contrary to global optimization methods in which the fine tuning of the numerous parameters is definitely a difficulty. Finally, there is no restriction regarding the type of array and pattern to be synthesized, since arbitrary array and any shaped beampattern can be handled.

\section{REFERENCES}

[1] M. I. Skolnik, "Nonuniform arrays," in Collin-Zucker Antenna Theory. New York: McGraw-Hill, 1969, ch. 6.

[2] R. E. Willey, "Space tapering of linear and planar arrays," IRE Trans. Antenna Propag., vol. 10, pp. 369-377, 1962.

[3] O. M. Bucci, M. D’Urso, T. Isernia, P. Angeletti, and G. Toso, "Deterministic synthesis of uniform amplitude sparse arrays via new density taper techniques," IEEE Trans. Antennas Propag., vol. 58, no. 6, pp. 1949-1958, Jun. 2010.

[4] R. L. Haupt, "Thinned arrays using genetic algorithms," IEEE Trans. Antennas Propag., vol. 42, no. 7, pp. 993-999, Jul. 1994.

[5] J. W. Hooker and J. K. Arora, "Optimal thinning levels in linear arrays," IEEE Antennas Wireless Propag. Lett., vol. 9, pp. 771-774, 2010.

[6] O. Quevedo-Teruel and E. Rajo-Iglesias, "Ant colony optimization in thinned array synthesis with minimum sidelobe level," IEEE Antennas Wireless Propag. Lett., vol. 5, pp. 349-352, 2006.

[7] A. Trucco, E. Omodei, and P. Repetto, "Synthesis of sparse planar arrays," Electron. Lett., vol. 33, no. 22, pp. 1834-1835, Oct. 1997.

[8] A. Trucco, "Weighting and thinning wide-band arrays by simulated annealing," Ultrasonics, vol. 40, pp. 485-489, 2002.

[9] D. G. Leeper, "Isophoric arrays - massively thinned phased arrays with well-controlled sidelobes," IEEE Trans. Antennas Propag., vol. 47, no. 12, pp. 1825-1835, Dec. 1999.

[10] G. Oliveri, M. Donelli, and A. Massa, "Linear array thinning exploiting almost difference sets," IEEE Trans. Antennas Propag., vol. 57, no. 12, pp. 3800-3812, Dec. 2009.

[11] R. M. Leahy and B. D. Jeffs, "On the design of maximally sparse beamforming arrays," IEEE Trans. Antennas Propag., vol. 39, no. 8, pp. 1178-1187, Aug. 1991.

[12] S. Holm, B. Elgetun, and G. Dahl, "Properties of the beampattern of weight- and layout- optimized sparse arrays," IEEE Trans. Ultrason. Ferroelect. Freq. Control, vol. 44, no. 5, pp. 983-991, Sep. 1997.

[13] Y. Liu, Z. Nie, and Q. H. Liu, "Reducing the number of elements in a linear antenna array by the Matrix Pencil Method," IEEE Trans. Antennas Propag., vol. 56, no. 9, pp. 2955-2962, Sep. 2008.

[14] Y. Liu, Z. Nie, and Q. H. Liu, "A new method for the synthesis of non-uniform linear arrays with shaped power patterns," Progr. Electromagn. Res., vol. 107, pp. 349-363, 2010.
[15] Y. Liu, Q. H. Liu, and Z. Nie, "Reducing the number of elements in the synthesis of shaped-beam patterns by the forward-backward matrix pencil method," IEEE Trans. Antennas Propag., vol. 58, no. 2, pp. 604-608, Feb. 2010

[16] T. Isernia, F. J. A. Pena, O. M. Bucci, M. D’Urso, J. F. Gomez, and J. A. Rodriguez, "A hybrid approach for the optimal synthesis of pencil beams through array antennas," IEEE Trans. Antennas Propag., vol. 52, no. 11, pp. 2912-2918, Nov. 2004.

[17] M. D'Urso and T. Isernia, "Solving some array synthesis by means of an effective hybrid approach," IEEE Trans. Antennas Propag., vol. 55, no. 3, pp. 750-759, Mar. 2007

[18] S. E. Nai, W. Ser, Z. L. Yu, and H. Chen, "Beampattern synthesis for linear and planar arrays with antenna selection by convex optimization," IEEE Trans. Antennas Propag., vol. 58, no. 12, pp. 3923-3930, Dec. 2010.

[19] M. S. Lobo, M. Fazel, and S. P. Boyd, "Portfolio optimization with linear and fixed transaction costs," Ann. Oper. Res., vol. 152, no. 1, pp. 341-365, Jul. 2007.

[20] E. J. Candès, M. B. Wakin, and S. P. Boyd, "Enhancing sparsity by reweighted $l_{1}$ minimization," J. Fourier Analy. Appl., vol. 14, pp. 877-905, Dec. 2008.

[21] J. J. Fuchs and B. Delyon, "Minimal $l_{1}$-norm reconstruction function for oversampled signals: Applications to time-delay estimation," IEEE Trans. Infor. Theory, vol. 46, no. 4, pp. 1666-1673, Jul. 2000.

[22] D. L. Donoho and X. Huo, "Uncertainty principles and ideal atomic decomposition," IEEE Trans. Infor. Theory, vol. 47, no. 7, pp. 2845-2862, Nov. 2001.

[23] A. Ben-Tal and A. S. Nemirovski, Lectures on Modern Convex Optimization: Analysis, Algorithms, and Engineering Applications. Philadelphia, PA: Society for Industrial and Applied Mathematics, MPS-SIAM, 2001.

[24] SeDuMi, [Online]. Available: http://sedumi.ie.lehigh.edu/

[25] A. Trucco, "Synthesizing asymmetric beam patterns," IEEE Trans. Oceanic Eng., vol. 25, no. 3, pp. 347-350, Jul. 2000.

[26] T. Isernia, O. M. Bucci, and N. Fiorentino, "Shaped beam antenna synthesis problems: Feasibility criteria and new strategies," J. Electromagn. Waves Applicat., vol. 12, pp. 103-138, 1998.

[27] R. S. Elliot and G. J. Stern, "A new technique for shaped beam synthesis of equispaced arrays," IEEE Trans. Antennas Propag., vol. 32, no. 10, pp. 1129-1133, Oct. 1984.

[28] H. L. Van Trees, Optimum Array Processing, Part IV of Detection, Estimation and Modulation Theory. New York: Wiley, 2002. 